\title{
Mental health and opioid addiction comorbidities among chronic pain patients
}

\author{
Bill Saunders ${ }^{1,3}$, Kevin Cevasco 2,1 \\ ${ }^{1}$ Watson Health, IBM, Cambridge, Massachusetts, United States, ${ }^{2}$ George Mason University, Fairfax Station, Virginia, United States, ${ }^{3}$ UNC Charlotte, \\ Charlotte, North Carolina, United States
}

\section{Objective}

Assessing mental health and opioid addiction comorbidities among chronic pain patients using a large longitudinal clinical, operational, and laboratory data set.

\section{Introduction}

The National Institute for Drug Abuse Report, Common Comorbidities with Substance Use Disorders, states there are "many individuals who develop substance use disorders (SUD) are also diagnosed with mental disorders, and vice versa." [1] Prescription opioids are amongst the most commonly used drugs that lead to illicit drug use [2]. Much of the data about the starting point of the prescription opioid addiction is in the patient health history and is recorded within the provider electronic health record and administrative systems.

\section{Description}

There are a variety of addiction and misuse risk screening tools available and selecting appropriate tools screening can be confusing for providers. Examples of common screening tools: Opioid Abuse Risk Screener (OARS), Opioid Risk Tool (ORT), Screener and Opioid Assessment for Patients with Pain (SOAPP), Current Opioid Misuse Measure (COMM), Diagnosis, Intractability, Risk, and Efficacy (DIRE). These opioid risk screening tools are interview based and vary in how they survey for psychosocial factors. The screening tools are useful, but are meant only to alert the provider to conduct further investigation [3]. Understanding how the comorbidities recorded in the patient's clinical interactions may help improve risk assessment investigations and ongoing monitoring programs. Studying the chronic pain patients' longitudinal clinical, operational, and laboratory records provides the basis for better study controls than those using population based on emergency department admission and mortality events.

\section{Methods}

The analysis leverages IBM's Explorys electronic health record (EHR) data, a large integrated source of real world clinical, operational and lab data across 39 large integrated delivery networks that span the continuum of care. In addition to demographic characteristics of drug abusers, we will describe common comorbidities of selected mental health diagnoses, examine codingrelated issues, distinguish chronic and episodic addiction and look for regional differences due to state/local level prescribing training and provider addiction awareness.

\section{References}

1. Abuse NI on D. Part 1: The Connection Between Substance Use Disorders and Mental Illness [Internet]. [cited 2018 Sep 29]. Available from: https://www.drugabuse.gov/publications/research-reports/commoncomorbidities-substance-use-disorders/part-1-connection-between-substance-use-disorders- mental-illness

2. Lankenau SE, Teti M, Silva K, Bloom JJ, Harocopos A, et al. 2012. Initiation into prescription opioid misuse amongst young injection drug users. Int J Drug Policy. 23(1), 37-44. PubMed https://doi.org/10.1016/j.drugpo.2011.05.014

3. Hudspeth RS. 2016. Safe Opioid Prescribing for Adults by Nurse Practitioners: Part 1. Patient History and Assessment Standards and Techniques. J Nurse Pract. 12(3), 141-48.

https://doi.org/10.1016/j.nurpra.2015.10.012 